\title{
Complex proximal humeral fractures (three or four part) with dislocation: outcome analysis of percutaneous reduction and external fixation
}

\begin{abstract}
Background: The complex fractures of the proximal humerus remain dilemmas far as treatment is concerned. Although better-quality techniques of internal fixation and prosthetic replacement are introduced effectively in the field of operative fracture care, management of complex fractures of the proximal humerus for head preserving modalities in injuries involving the humeral head remains to be a major factor concerning the trauma surgeon.

Materials \& Methods: Thirty nine patients with complex three or four part proximal fracture of humerus with dislocation of humeral head were treated and analyzed for results of percutaneous reduction and external fixation. All Patients were subjected to careful closed reduction under general anesthesia with optimal relaxation and fluoroscopic control followed by percutaneous external fixation of proximal humerus in optimal position. The patients were evaluated clinically and radiologically for a period till union or 10 months, whichever was earlier. One patient had an incomplete brachial plexus injury, which was yet to recover completely at the end of last follow up 10 months, with acceptable hand, elbow and shoulder function.
\end{abstract}

Results: Percutaneous reduction \& external fixation to treat complex fractures of the proximal humerus produces good results, if correct re-position and biological principles are respected. Most of the patients were pain-free and the shoulders functioned well despite the severity of shoulder injury.

Conclusions: Percutaneous reduction and external fixation is a reasonable and logical approach to treat complex proximal humeral head fractures, rather than conservative treatment and before hemi-arthroplasty by prosthesis is considered, in view of the acceptable results obtained. Our study shows that complex fractures of the proximal humerus should be treated initially by head preserving procedures. The probable revascularization of the humeral head occurs by creeping substitution as the shoulder is non weight bearing joint.

Keywords: Complex fractures, Constant Score, External fixation, Proximal Humeral fractures, Percutaneous Reduction
Volume 6 Issue 5 - 2016

\author{
Nikose S,' Khan S, ${ }^{2}$ Mundhada G, ${ }^{2}$ Singh P, I \\ Gudhe $M,{ }^{2}$ Nikose D, ${ }^{3}$ Arora $M^{2}$ \\ 'Department of orthopedics, Datta Meghe Institute of Medical \\ Sciences, India \\ ${ }^{2}$ Senior Resident, Datta Meghe Institute of Medical Sciences, \\ India
}

${ }^{3}$ Medical Student, NKP Salve Institute of Medical Sciences, India

Correspondence: Sunil Nikose, Department of Orthopaedics, Datta Meghe Institute of Medical Sciences, Sawangi(Meghe), Wardha, India - 44200I, Tel 7|5228770।

Email sunilnikose@gmail.com

Received: December 0I, 2015 | Published: December 08, 2016

\section{Introduction}

The Proximal Humerus fractures most commonly effects the elderly population and elderly women with osteoporosis. These fractures accounts for a total of $4 \%$ to $5 \%$ of all the fractures. ${ }^{1}$ The majority $(85 \%)$ of these fractures show minimal or no displacement and can be treated successfully with conservative treatment and early mobilization. ${ }^{2,3,4}$ In contrast, conservative management of displaced fractures of proximal humerus often results in mal union and poor shoulder function. In this subset of individuals surgical treatment is recommended. ${ }^{5}$ The widely accepted treatment of severely comminuted intra-articular proximal humeral fractures is hemi-arthroplasty or total shoulder arthroplasty. ${ }^{6,7}$ Several surgical treatment options, such as closed reduction and percutaneous fixation. ${ }^{8}$ open reduction and internal fixation with a variety of constructs. ${ }^{9-13}$ proximal humeral intra medullary nailing. ${ }^{14}$ and shoulder arthroplasty. ${ }^{15}$ have been used with relatively good outcomes.

There are very few reported studies in literature regarding external fixation of displaced proximal humeral fractures.5,16-23 Compared with open reduction and osteo-synthesis, external fixation techniques for displaced proximal humeral fractures avoid dissection and stripping of the soft tissues, leads to higher union rates, has a lower incidence of avascular necrosis, prevention of normal scapula humeral relation at its border and speedy rehabilitation.16,17 There are however, conflicting reports from different studies regarding use of external fixation as treatment modality for displaced proximal humeral fractures. ${ }^{5,17-23}$ Many studies in literature reports good or excellent results and less complication compared with open techniques. ${ }^{20-23}$ whereas some other studies reports state that external fixation and stabilization of proximal humeral fractures is technically demanding and can be used for two part 2-part proximal humeral fractures. External fixation also might lead to acceptance of less than optimal reduction as far as fracture stability is concerned, especially in elderly population with poor bone stock (osteoporosis). ${ }^{5,17-19}$ In our study we aimed to analyse the results of closed reduction and external fixation for displaced three or four part fractures involving humeral head and associated with dislocated humeral head. The rationale is that closed reduction and external fixation for displaced proximal humeral fractures along with dislocation would prevent the replacement of head of humerus and is safe procedure, with good clinical results and acceptable minimal complications.

\section{Materials and methods}

Thirty nine patients with displaced three or four-part fractures of proximal humerus along with dislocated humeral head were included in this study and were treated by closed reduction of fracture and dislocation along with external fixation of proximal humerus. The study was conducted between January 2010 and January 2014. 
There were 16 women and 23 men with an age range of $20 \pm 59$ years (Mean 49 years). All patients were evaluated clinically and radiologically postoperatively for a period till union and for one year. The mechanism of injury was moderate to severe in most of our patients and the primary reason being road traffic accident or was a fall while at work. All patients had informed and written consent obtained for the purpose of the study. The patients were informed about the benefits of external fixation and stabilization which included less invasiveness of the surgical procedure, minimal or less soft tissue dissection and almost no soft tissue stripping and the possible complications of external fixation which included loss of fracture reduction requiring more extensive surgery, pins migration and pintract infection; discomfort due to external fixator during the course of treatment. ${ }^{5,8,16-23}$ The present study was approved by ethical committee of institution. Fractures of proximal humerus with dislocation were graded as per Neer's classification. ${ }^{24}$ which includes 2 part, 3 part and 4 part fractures and fracture-dislocations, based on severity of fracture pattern. According to Neer. ${ }^{24}$ more than $1 \mathrm{~cm}$ displacement of a fracture or angulation more than $45^{\circ} \mathrm{C}$ is significant requiring surgical management to preserve shoulder function. ${ }^{24}$ All patients in our series had three or four part fractures along with dislocated humeral head according to Neer's classification.

$100 \%$ of our patients had closed injury of proximal humerus, with the exception of minor abrasions; however, there shoulder and upper arm were grossly swollen, ecchymosed along with severe pain in range of 8/10 of VAS (Visual Analogue Score). Between January 2010 and January 2014 we had one hundred and eighty four (184) patients comprising of all proximal humeral fractures. One hundred and thirty patients had minor non displaced or minimally displaced greater tuberosity or surgical neck fractures which were managed by simple arm sling application treatment till pain subsidence. Thirteen patients had Neer's type two or three upper humerus fractures without dislocation of head of humerus. Another two patients of pathological proximal humerus fractures and were treated accordingly. The remaining 39 patients had displaced three or four-part fractures of proximal humerus along with dislocation of head of humerus and were treated by closed reduction, percutaneous external stabilization of proximal humerus and included in our study. The external fixator device was safely removed when there were signs of clinical or radiological union (subsidence of pain, callus formation). Shoulder range of movement exercises were started as soon as the pain allowed. Instructions were provided for the care for pin sites. Patients were followed up as necessary and at 6 week and 3,6,10 and at 12 months. Shoulder function was assessed using Constant Shoulder Score (C Constant and A Murley.) at each follow up which is both subjective and objective and consists of 100 points (Pain-15, Activity of Daily Living- 20 , Movements- 40 Strength-25) (Figures 1-8).

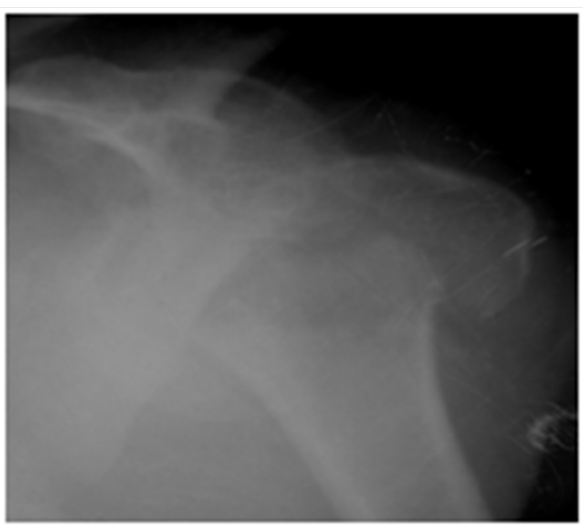

Figure I Pre-operative.

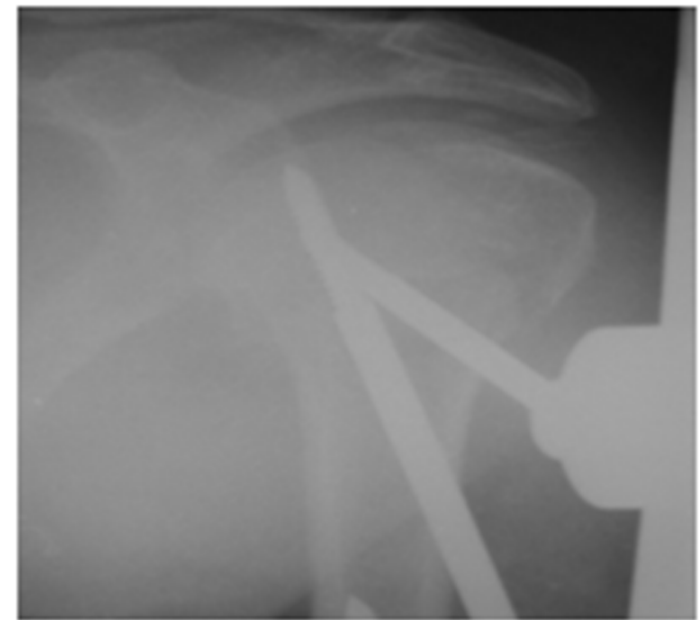

Figure 2 Immediate post-operative- Since this was initial of our patients, the greater tuberosity was not considered for fixation; however in our later cases, greater tuberosity was fixed as a rule.

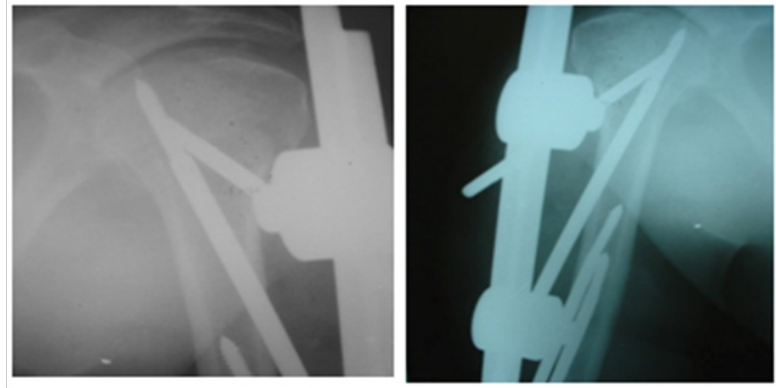

Figure 36 Weeks post operative-Prior to fixator removal.

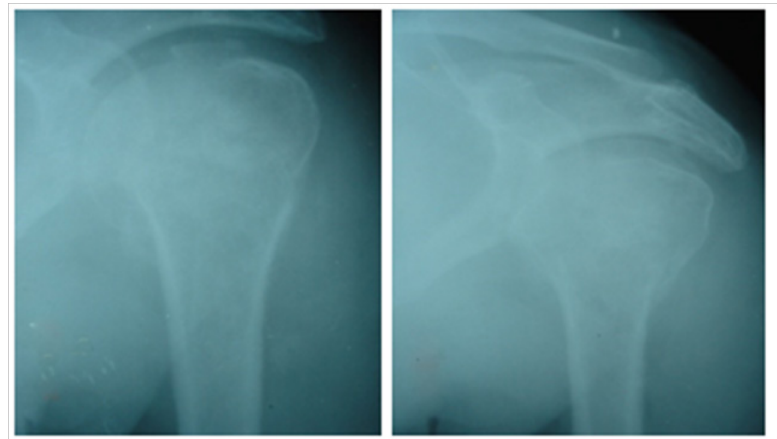

Figure 46 months postoperative film-The humeral head appears contained in glenoid and the patient had reasonable shoulder function. The head of humerus did not appear avascular at 6 months.
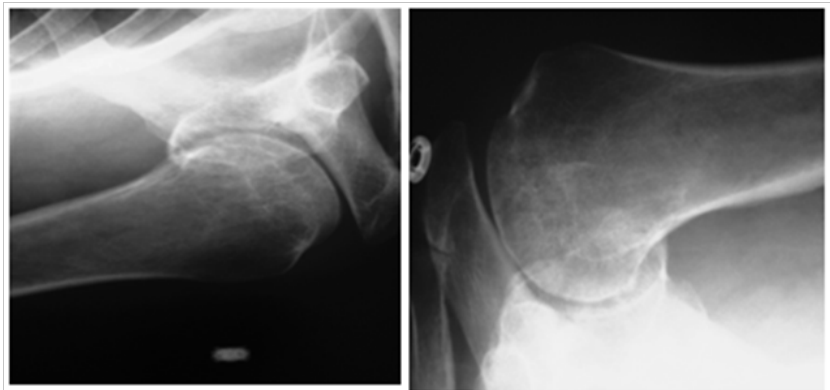

Figure 5 I4 months postoperative-The humeral head appeared contained with post traumatic arthritis due to avascular necrosis. Active abduction was about 90 degrees at shoulder. 

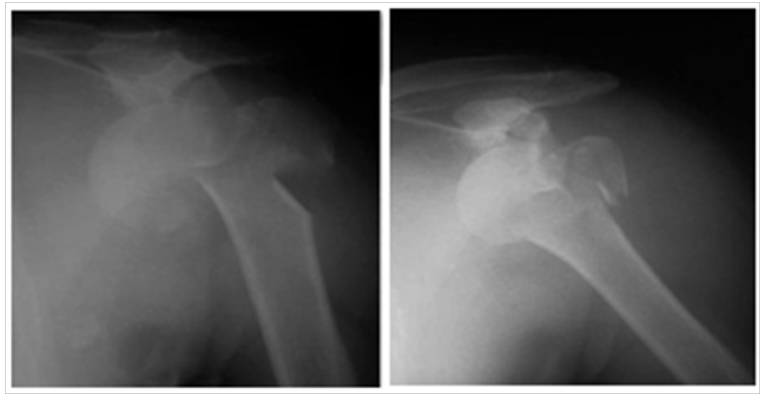

Figure 6 Pre- operative four part fracture dislocation.
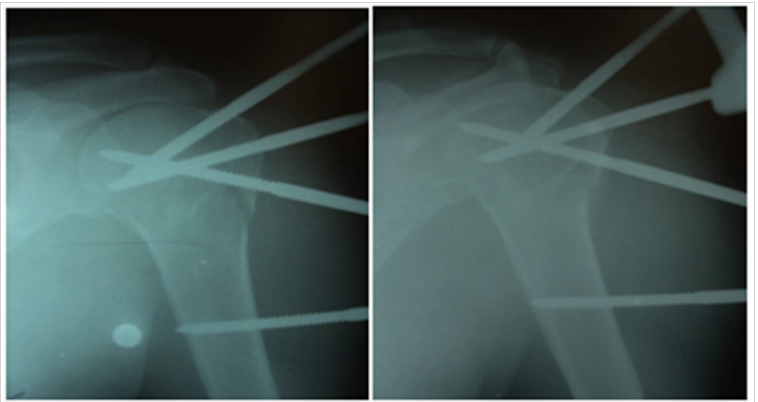

Figure 7 Immediate postoperative films showing congruous reduction, stabilized greater tuberosity and contained head of humerus.

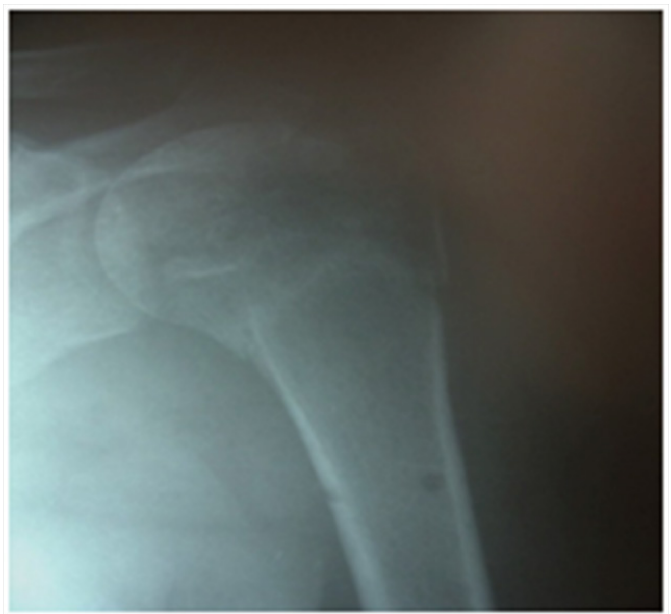

Figure 86 months postoperative films-good contained head of humerus, no signs of avascular necrosis and patient had excellent shoulder function.

\section{Surgical Technique}

With patient under general anesthesia on a standard operating table with fluoroscopy control, Using fluoroscopy control, trial of humeral head reduction was performed and followed by reduction of the three or four-part fracture with intra operative manipulation using Kirschner wires. A Steinman pin in distal fragment was used as a joystick for traction as well as reduction. The reduction was accepted following Neer's criteria for reduction. ${ }^{24}$ All the patients achieved acceptable closed reduction of their fracture. Two or three pins were inserted in proximal fragment and two pins were inserted in the humeral shaft and the fixator was applied (Figure 9).

\section{Results}

The interval between injury and surgery was around 4.5 (range $0-9)$ days; 33 (84.61\%) patients were operated in a weeks' time. The duration of external fixation removal was 48 (range, 39-56) days. The time to radiological union took almost 12 (range 9-14) weeks. The follow-up period was 14 months (range, 12-18). Reduction was good and maintained (Almost anatomical reposition of fragments) in 22 (56.41\%) patients and fair in $17(43.58 \%)$ patients with a reasonable alignment of opposing fracture fragments. 7 (17.94\%) patients had pin tract infections during course of external fixator application and were managed appropriately till healing. $2(5.12 \%)$ patients developed avascular necrosis of head of humerus starting after 6 months of injury. The function of shoulder was done according to the Shoulder Constant Score (C Constant and Murley). The shoulder function was found excellent in $16(41.02 \%)$ patients, good in $14(35.89 \%)$, moderate in $5(12.82 \%)$ and poor in $4(10.25 \%)$ patients. One $(2.56 \%)$ patient had early inferior subluxation of gleno humeral joint which required frame readjustment despite initial good reduction. This was probably due to complex nature of the four part fracture dislocation (Figure 10).

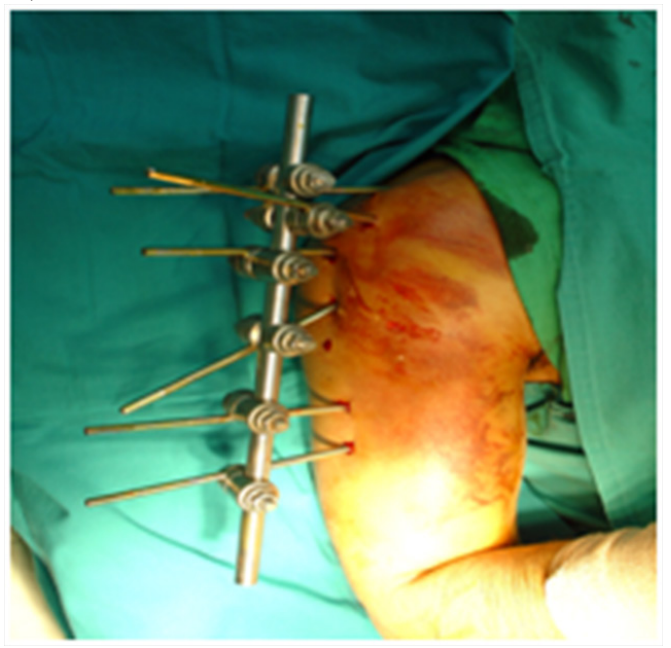

Figure 9 Clinical picture of application of fixator - Note the severity of injury- with swelling and ecchymosis along with considerable soft tissue injury.

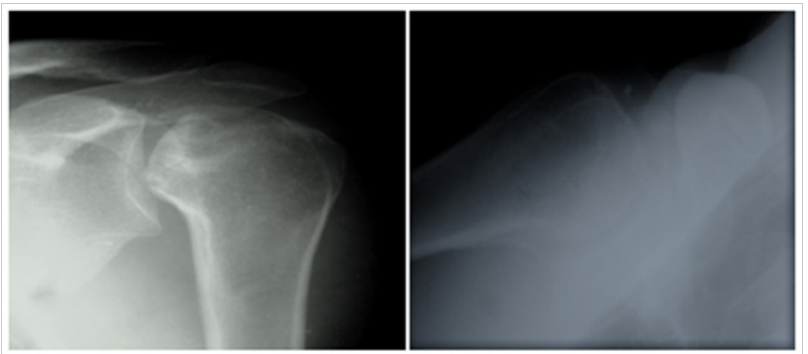

Figure 10 The patient with post traumatic avascular necrosis of femoral head - There was collapse of humeral head along with poor shoulder function.

\section{Discussion}

In developing countries like India, the pattern of fracture varies as compared to developed countries, due to the way of working and mode of trauma sustained. Our patients were younger when we compared to the other reports in the available literature., ${ }^{3,12}$ (our study49 versus other studies-56-61 years). Since the injury sustained was in a much younger age group, men were predominantly involved in our study, compared to predominance of women (61-89\%) in reported literature due to the age difference at which the injury is sustained (osteoporosis and fragility fractures due to trivial fall). ${ }^{13,14}$ The patients in our study were heavy workers and/or labourers and their outcome would be disproportionate due to undue loads placed on shoulder region. Twenty nine (74.35\%) of these injuries in our series were due to high-energy trauma sustained, compared to the 
other studies where almost $89 \%$ caused by moderate trauma and $93 \%$ following trivial trauma involving activities of daily living, thus giving a higher proportion of osteoporotic fractures around shoulder. ${ }^{4,15}$ Our patients had a mean radiological union time of 12 weeks, as compared to 15.2 weeks (3.8 months) in studies where open reduction along with internal fixation or a combination of internal and external fixation was employed. ${ }^{16}$ Thirty $(76.92 \%)$ of our patients had excellent or good results when the shoulder was evaluated on the basis of pain, objectively and subjectively in comparison to $40 \%$ after close manipulation in other studies, $73 \%$ after use of external stabilisation after reduction and 52\% when open reduction and osteo-synthesis procedures were employed. ${ }^{9,17}$ Since our patients were much younger and anticipated demands on the shoulder were higher the results may be disproportionate. There were no controls in our study for obvious reasons and the sample size of individuals sustaining this type of injury was moderate, considering the uncommonness of this type of complex injury. In a country like India, inclusive of south Asia, these complex fractures are usually treated with limited resources in best of the institutes and external fixation/stabilisation can be the choice of treatment for displaced and complex proximal humerus fractures, ${ }^{18}$ because it doesn't further compromises the blood supply of the head of humerus and doesn't further traumatises the soft tissues, enabling early mobilisation of the shoulder which in turn causes reasonable shoulder function and healing in optimal position despite sometimes pin loosening. We made sure that the reduction of greater tuberosity is achievable and maintained, because Malunion and nonunion of greater tuberosity leads to poor outcome due to rotator cuff dysfunction. ${ }^{25}$ This is the probable reason that the patients showed better results in those who had accurate/good reduction of the greater tuberosity.

Complications of this complex injury are common despite employing best of the open treatment and even with non-operative management. Those leading to poor patient outcome is avascular necrosis, stiffness of the shoulder and Malunion. ${ }^{26-28} 2(5.12 \%)$ of our patients developed avascular necrosis of humerus head. One patient was moderately pain free and another patient had constant and prolonged pain, which caused stiffness of shoulder, affecting activity of daily living after first six months of treatment, which gradually improved to some extent without any active surgical intervention. These two patients with humeral head osteonecrosis however showed clinical and radiological improvement after six months, suggesting a fact that revascularization of head of humerus might be due to creeping substitution, especially since shoulder is a non-weight bearing joint. Open surgical techniques, reduces the incidence of Malunion, but increases chances of surgical site infection, avascular necrosis of humeral head due to stripping, iatrogenic neurologic or vascular injury and implant or hardware failure. ${ }^{29-32}$ We had pin track infection in seven $(17.94 \%)$ patients, which was considered high, but the fixator was kept because it offered stability to fracture fragments and patients were treated appropriately. The benefits of closed reduction and external fixation of this complex proximal humerus fracture by external fixation and stabilization apparently overweigh these minor undesirable complications during the course of treatment.

\section{Conclusion}

External fixation preserves the remaining residual vascularity of these fracture fragments and enables quick rehabilitation and offers early mobilization, thus accomplishing safe healing with a good function. It is effective for management of complex humeral dislocation with displaced proximal humerus fractures and hence it is concluded that closed reduction and external fixation/stabilization is indicated initially rather than head sacrificing procedures or conservative management, in view of the good results obtained after the management with external stabilization. These data show that complex fractures of the proximal humerus should be treated by head preserving surgeries especially in young patients having high demand on shoulder due to their way of working. The risk of avascular necrosis is mild and since the shoulder is a non-weight bearing joint, painful symptoms appear late and could be self limiting sometimes.

\section{Acknowledgments}

None.

\section{Conflicts of interest}

None.

\section{References}

1. Handoll $\mathrm{HH}$, Ollivere BJ, Rollins KE Interventions for treating proximal humeral fractures in adults. Cochrane Database Syst Rev. 2012;12:CD000434.

2. Brown CMC, Garg A, McQueen MM The epidemiology of proximal humeral fractures. Acta Orthopaedica Scandinavica. 2001;72(4):365-371.

3. Clifford PC Fractures of the neck of the humerus: a review of the late results. Injury. 1980;12(2):91-95.

4. Kristiansen B, Christensen SW Proximal humeral fractures. Late results in relation to classification and treatment. Acta Orthop Scand. 1987;58(2):124-127.

5. Calvo E, De Miguel I, De La Cruz JJ et al. Percutaneous fixation of proximal humeral fractures: indications based on the correlation between clinical and radiographic results. J Shoulder Elbow Surg. 2007;16(6):774-781.

6. Kralinger F, Schwaiger $\mathrm{R}$, Wambacher $\mathrm{M}$ et al. Outcome after primary hemiarthroplasty for fracture of the head of the humerus. A retrospective multicentre study of 167 patients. J Bone Joint Surg Br. 2004;86(2):217-219.

7. Neer CS Displaced proximal humeral fractures. II. Treatment of three-part and four-part displacement. J Bone Joint Surg Am. 1970;52(6):1090-1103.

8. Dolfi H, Darrick TS, Marie PJ et al. Percutaneous fixation of proximal humeral fractures. Clinical Orthopaedics \& Related Research. 2000;375:97-104.

9. Cornell CN, Levine D, Pagnani MJ Internal fixation of proximal humerus fractures using the screw-tension band technique. J Orthop Trauma. 1994;8(1):23-27.

10. Gerber C, Werner CM, Vienne P Internal fixation of complex fractures of the proximal humerus. J Bone Joint Surg Br. 2004;86(6):848-855.

11. Drosdowech DS, Faber KJ, Athwal GS Open reduction and internal fixation of proximal humerus fractures. Orthopedic Clinics of North America. 2008;39(4):429-439.

12. Park MC, Murthi AM, Roth NS et al. Two-part and three-part fractures of the proximal humerus treated with suture fixation. J Orthop Trauma. 2003;17(5):319-325.

13. Kristiansen B, Christensen SW Plate fixation of proximal humeral fractures. Acta Orthop Scand. 1986;57(4):320-323.

14. Young AA, Hughes JS Locked intramedullary nailing for treatment of displaced proximal humerus fractures. Orthop Clin North Am. 2008;39(4):417-428.

15. Willems WJ, Lim TE Neer arthroplasty for humeral fracture. Acta Orthop Scand. 1985;56(5):394-395.

16. Keener JD, Parsons BO, Flatow EL et al. Outcomes after percutaneous reduction and fixation of proximal humeral fractures. J Shoulder Elbow Surg. 2007;16(3):330-338. 
17. Fenichel I, Oran A, Burstein $G$ Percutaneous pinning using threaded pins as a treatment option for unstable two and three-part fractures of the proximal humerus: a retrospective study. Int Orthop. 2006;30(3):153-157.

18. Brunner A, Weller K, Thormann S et al. Closed reduction and minimally invasive percutaneous fixation of proximal humerus fractures using the Humerusbloc. J Orthop Trauma. 2010;24(7):407-413.

19. Resch H, Hübner C, Schwaiger R Minimally invasive reduction and osteosynthesis of articular fractures of the humeral head. Injury. 2001;32(Suppl 1):SA25-SA32.

20. Kristiansen B, Kofoed H External fixation of displaced fractures of the proximal humerus: technique and preliminary results. J Bone Joint Surg Br. 1987;69(4):643-646.

21. Kristiansen B, Kofoed H Transcutaneous reduction and external fixation of displaced fractures of the proximal humerus: a controlled clinical trial. J Bone Joint Surg Br. 1988;70(5):821-824.

22. Monga P, Verma R, Sharma VK Closed reduction and external fixation for displaced proximal humeral fractures. J Orthop Surg (Hong Kong) 2009;17(2):142-145.

23. Martin C, Guillen M, Lopez G Treatment of 2 and 3-part fractures of the proximal humerus using external fixation: a retrospective evaluation of 62 patients. Acta Orthopaedica. 2006;77(2):275-278.

24. Neer CS Displaced proximal humeral fractures. I. Classification and evaluation. J Bone Joint Surg Am. 1970;52(6):1077-1089.

25. Boileau P, Krishnan SG, Tinsi L et al. Tuberosity malposition and migration: reasons for poor outcomes after hemiarthroplasty for displaced fractures of the proximal humerus. J Shoulder Elbow Surg. 2002;11(5):401-412.
26. Poeze M, Lenssen AF, Van Empel JM et al. Conservative management of proximal humeral fractures: can poor functional outcome be related to standard transscapular radiographic evaluation? J Shoulder Elbow Surg. 2010;19(2):273-281

27. Tejwani NC, Liporace F, Walsh M et al. Functional outcome following one-part proximal humeral fractures: a prospective study. J Shoulder Elbow Surg. 2008;17(2):216-219.

28. Hanson B, Neidenbach P, De Boer P et al. Functional outcomes after nonoperative management of fractures of the proximal humerus. $J$ Shoulder Elbow Surg. 2009;18(4):612-621.

29. Bogner R, Hübner C, Matis N et al. Minimally-invasive treatment of three and four-part fractures of the proximal humerus in elderly patients. J Bone Joint Surg Br. 2008;90(12):1602-1607.

30. Nho SJ, Brophy RH, Barker JU et al. Management of proximal humeral fractures based on current literature. J Bone Joint Surg Am 89 Suppl. 2007;3:44-58.

31. Brunner F, Sommer C, Bahrs $\mathrm{C}$ et al. Open reduction and internal fixation of proximal humerus fractures using a proximal humeral locked plate: a prospective multicenter analysis. J Orthop Trauma. 2009;23(3):163-172.

32. Südkamp N, Bayer J, Hepp P et al. Open reduction and internal fixation of proximal humeral fractures with use of the locking proximal humerus plate. Results of a prospective, multicenter, observational study. $J$ Bone Joint Surg Am. 2009;91(6):1320-1328. 